Disponível em:

http://editora.unoesc.edu.br/index.php/race

Race, Joaçaba, v. 14, n. 1, p. 121-142, jan./abr. 2015

\title{
APLICAÇÃO DA FRONTEIRA EFICIENTE POR MEIO DAS TÉCNICAS DE BOOTSTRAPPING E MONTE CARLO: UMA PARALELIZAÇÃO ENTRE BM\&FBOVESPA E NYSE A PARTIR DAS PRINCIPAIS ADRS BRASILEIRAS
}

\section{Application of efficient frontier through bootstrapping and Monte Carlo techniques: a parallelization between BMEFBovespa and Nyse from major brazilian ADRs}

Carolina Magda da Silva Roma

E-mail: carolina.magda.adm@gmail.com Mestre em Administração pela Universidade Federal de Pernambuco; Doutoranda no Centro de Pós-graduação e Pesquisas em Administração/Finanças na Universidade Federal de Minas Gerais.

Robert Aldo Iquiapaza E-mail: riquiapaza@gmail.com Doutor e Mestre em Administração/Finanças pela Universidade Federal de Minas

Gerais; Professor Adjunto da Universidade Federal de Minas Gerais; Avenida Presidente Antonio Carlos, 6627, Pampulha, 31271-970, Belo Horizonte, Minas Gerais, Brasil.

Bruno Pérez Ferreira E-mail: brunoperez.bh@gmail.com Doutor e Mestre em Administração/Finanças pela Universidade Federal de Minas Gerais; Professor Adjunto da Universidade Federal de Minas Gerais. 


\section{Resumo}

Neste artigo é revisitada a área de gestão de investimento no artigo de Markowitz (1952) que definiu formalmente o retorno de um investimento e o risco, inserindo no cômputo deste último a covariância, isto é, a forma como os ativos se movimentam um em relação ao outro. Com tais definições realizadas, Markowitz (1952) apresentou a fronteira eficiente como aquele conjunto de investimento que apresenta a melhor relação retorno versus risco, o qual os investidores podem utilizar para balizar seus investimentos. Dessa forma, a presente pesquisa objetivou construir, a partir de dados históricos mensais relativos ao período entre fevereiro de 2010 e abril de 2013, a fronteira eficiente utilizando três metodologias diferentes de dados de entrada, que foram a maneira tradicional baseada na série histórica, por bootstrapping e Simulação de Monte Carlo, como também a obtenção do Índice de Sharpe (IS) para identificar a possível superioridade de algum dos métodos. Para esse fim, foi selecionada uma amostra composta por 10 companhias brasileiras emissoras de American Depositary Receipts (ADRs) e classificadas como Top Components do Dow Jones Brazil Titans ADR Index (BR 20). Os principais resultados evidenciaram que pelo método de bootstrapping comparado com a Simulação de Monte Carlo foi possível rejeitar a hipótese nula de que produzam ISs iguais, porém, não é possível ser feita a mesma afirmação analisando por bootstrapping em relação à metodologia tradicional.

Palavras-chave: Fronteira eficiente. Dados históricos. Bootstrapping. Simulação de Monte Carlo. Índice de Sharpe.

\section{Application of efficient frontier through bootstrapping and Monte Carlo techniques: a parallelization between BMEFBovespa and Nyse from major brazilian ADRs}

\section{Abstract}

In this paper we revisited the area of investment management in the article by Markowitz (1952) who formally defined the return of an investment and the risk by entering in the computation of the latter the covariance, i.e., how the assets move in relation to each other. With these definitions made, Markowitz (1952) introduced the efficient frontier as one set of investment that offers the best relation return versus risk, which investors can use to mark their investments. Thus, the present study aimed to build, from monthly historical data for the period between February 2010 to April 2013, the efficient frontier using three different methods of input data, which were the traditional way based on historical series, bootstrapping and Monte Carlo Simulation, but also to obtain Sharpe Index (SI) to identify the superiority of one of the possible methods. To this end, we selected a sample of ten Brazilian companies that are issuers of American Depositary Receipts (ADRs) and are classified as Top Components of the Dow Jones Brazil Titans ADR Index (BR 20). The main results showed that the method of bootstrapping compared with Monte Carlo Simulation was possible to reject the null hypothesis that produce SI equal, but cannot be made the same statement analyzing bootstrapping compared to a traditional method.

Keywords: Efficient frontier. Historical data. Bootstrapping. Monte Carlo Simulation. Sharpe Index. 


\section{INTRODUÇÃO}

Uma das preocupações de qualquer investidor pessoa física ou institucional está em como melhor gerir seus recursos de forma a otimizar tempo, recursos e maximizar a riqueza. Para tanto, o investidor busca papéis que possam lhe render um retorno aceitável considerando o nível de risco que está disposto a assumir. Uma regra básica dentro desse trade-off e que existe não somente na área de investimentos é que quanto maior o retorno exigido, mais risco terá que ser incorrido ou, conforme o ganhador do prêmio Nobel de Economia em 1976, "[...] there's no such thing as a free lunch." (FRIEDMAN, 1975). Essa é uma relação muito clara dentro do mercado de capitais, no qual as companhias se dirigem de forma a levantar recursos e os investidores e especuladores transacionam tais papéis visando à obtenção de ganhos e considerando o risco assumido.

A área de finanças tal qual é conhecida há algum tempo, que faz uso de metodologias objetivas e baseadas em modelos matemáticos para a gestão de investimentos, foi iniciada propriamente no artigo de Markowitz (1952), que iniciou a Moderna Teoria de Finanças. O autor estabeleceu que o retorno de um portfólio é atribuído pela média ponderada entre a participação dos ativos na carteira e o retorno de cada ativo. Porém, essa simples ponderação não é suficiente para mensurar o risco. A grande contribuição de Markowitz (1952) foi inserir no cálculo do risco de um ativo a covariância, isto é, a maneira como os ativos se movimentam um em relação ao outro. Então, o atual investidor que se baseava apenas no empirismo, pode passar a fazer uso de uma abordagem objetiva para identificar que peso atribuir a cada ativo em sua carteira.

A partir das variáveis retorno e risco, pode-se construir a fronteira eficiente que, conforme proposto por Markowitz (1952), é aquela que representa a melhor opção de retorno para determinado nível de risco ou, ainda, para determinado nível de risco não há um retorno maior que possa ser alcançado. Eis, então, o objetivo de cada investidor que busca maximizar sua função utilidade $(U=f(E(r), \sigma)$. Em outras palavras, a fronteira eficiente proposta pelo autor está fundamentada puramente na análise do retorno (médio) e risco (variância) de uma carteira de ativos.

Para Bernstein (1997) é o domínio do risco que separa os tempos modernos e o passado. Assim, a criação, o teste e o aperfeiçoamento de variadas técnicas que objetivam contribuir para a literatura e visam proporcionar estimativas mais acuradas de retorno e risco para o investidor pautar suas escolhas são sempre necessários. 
É nesse escopo que o presente trabalho se insere. Partindo da contribuição de Markowitz (1952) com os conceitos de retorno e risco, buscou-se construir a fronteira eficiente utilizando observações a partir de três métodos diferentes: fronteira tradicional de média-variância (MV) com dados históricos; fronteiras simuladas pelo método de bootstrapping (reamostragem para um tamanho igual à matriz original e outro para o dobro do número de observações) e Simulação de Monte Carlo comparar o resultado visando identificar se há superioridade de alguma delas diante das demais se baseando no teste t aplicado ao Índice de Sharpe (1966). Este Índice é um indicador bastante utilizado pelo mercado para acompanhar o desempenho da carteira e avaliar a performance dos gestores de fundos de investimento. Ainda, como amostra foram usados 10 American Depositary Receipts (ADRs) consideradas Top Components do índice Dow Jones Brazil Titans ADR Index (BR 20), o qual representa as companhias brasileiras mais negociadas no mercado americano, para o período de fevereiro de 2010 a abril de 2013, totalizando 39 observações mensais.

Como justificativa, pode-se apresentar a contribuição para a área de investimento comparando três diferentes metodologias que podem ser utilizadas pelo investidor no momento de selecionar os dados de input para estruturar a fronteira eficiente, isto é, aquela que representa a melhor oportunidade de investimento considerando seu perfil e por verificar de forma objetiva a relação entre elas.

O trabalho está estruturado da seguinte forma: na seção 2 são apresentados os principais conceitos dentro de teoria de carteiras e a utilização dos métodos alternativos para gerar as variáveis de input para montar a fronteira eficiente. $\mathrm{Na}$ seção 3, encontram-se os procedimentos metodológicos adotados. Na seção 4, são apresentados e discutidos os principais resultados da pesquisa e, por último é realizada a conclusão do trabalho.

\section{REFERENCIAL TEÓRICO}

\subsection{A FRONTEIRA EFICIENTE}

Já se passaram 62 anos desde que o artigo de Harry Markowitz foi publicado no The Journal of Finance, sob o título Portfólio Selection, que veio a revolucionar o campo de finanças. Tal campo, a partir das décadas de 1950 e 1960, passou por uma transformação teórico-metodológica se afastando da decisão pautada na base empírica e se firmando como um campo de caráter normativo que é encontrado no 
paradigma positivista funcionalista (IQUIAPAZA; AMARAL; BRESSAN, 2009). O trabalho de Markowitz (1952) fundamenta o que é denominado Moderna Teoria de Finanças, pois contribuiu apontando para uma mudança de rumo nos estudos relacionados à área que, conforme explicitado, possuíam um viés mais prático, para uma abordagem objetiva, apoiando-se, por exemplo, na matemática.

O trabalho de Markowitz (1952) estabeleceu como um investidor considerado racional, uma vez que suas formulações estão atreladas à teoria de utilidade esperada, poderia eficientemente otimizar um portfólio para alocar seus recursos. Para tanto, há que se valer do trade-off retorno versus risco, assumindo que o investidor, cujo comportamento está pautado na racionalidade, sempre busca maximizar o retorno possível e minimizar o risco ao qual está exposto analisando $N$ ativos. A exemplo disso, tem-se estudos como os que buscam fornecer estimativas para apoiar a tomada de decisão (a exemplo das medidas de retorno ajustadas ao risco, tal qual o índice de Sharpe e o índice de Treynor, formulados por Sharpe (1966) e Treynor (1965), respectivamente, e a variável prêmio de risco nos estudos de apreçamento de ativos, a exemplo do Capital Asset Pricing Model de Sharpe (1964), Lintner (1965) e Mossin (1966), entre outros. O método proposto por Markowitz (1952) para computar o retorno de um ativo e do risco (variância) é mostrado a seguir na sua forma matricial:

$$
\begin{gathered}
E(r)=w^{\prime} r \\
\sigma^{2}=w^{\prime} C w
\end{gathered}
$$

Sujeito a

$$
\begin{gathered}
\sum_{i=1}^{n} w=1 \\
w_{i} \geq 0 \quad \forall i=1,2, \ldots, N
\end{gathered}
$$

Em que ${ }^{w}$ representa o vetor de pesos de cada ativo a ser investido na carteira, $r$ representa o vetor retorno de cada ativo individual e $C_{\text {é a matriz de }}$ covariância amostral. Igualmente, pode-se verificar que o problema de otimização é restrito a apenas participações positivas, isto é, restringe vendas a descoberto, e que não haja ativos com peso nulo. Assim, três observações acerca do trabalho de Markowitz (1952) merecem destaque: primeira, o método apresentado é o 
de média-variância (MV), pois está baseado no parâmetro $\left(\mu, \sigma^{2}\right)$; segunda, no referido trabalho, somente foram considerados ativos de risco; e, terceira, a maior contribuição foi inserir a relevância da covariância para computar o risco da carteira, pois a forma como os ativos se movimentam um em relação ao outro permite colocar em jogo a relevância da diversificação no portfólio, que influencia diretamente na análise do trade-off retorno versus risco, pois quanto mais a carteira for diversificada, isto é, quanto mais os ativos forem negativamente correlacionados, mais proteção para o portfólio do investidor em movimentos contrários no mercado.

Ainda, Markowitz (1952) designou o conjunto eficiente de pontos (retorno, risco) como a fronteira eficiente. Conjunto eficiente, em outras palavras, significa que, para um determinado nível de risco, não há maior retorno ou que, para determinado nível de risco, não há menor risco. Para a construção da fronteira, calcula-se o retorno médio de cada ativo e a matriz de covariância amostral para que seja possível construir o portfólio de mínima variância (PMV), aquele com menor risco possível, e o portfólio tangente $(\mathrm{PT})$, isto é, aquele que apresenta o maior índice de Sharpe ou ainda aquele que maximiza o grau de inclinação, conforme apontado a seguir:

$$
\begin{aligned}
& P V M=C^{-1} \cdot 1 \\
& P T=C^{-1} \cdot P R
\end{aligned}
$$

Em que:

$P V M=$ portfólio de variância mínima;

$C^{-1}=$ matriz inversa de covariâncias dos retornos;

$P T=$ portfólio tangente;

$P R=$ prêmio de risco ou diferença entre o retorno de um determinado ativo considerado de risco e a proxy do ativo livre de risco.

Ainda, parte-se do pressuposto de que: para o PVM, as covariâncias de cada um dos ativos com o PVM são as mesmas para todos os ativos. Por exemplo, considerando três ativos na carteira denominados $k, n$ e $i$, o enunciado afirma que $\sigma_{k, P V M}=\sigma_{n, P V M}=\sigma_{i, P V M}$, sendo, então, igualado a um valor arbitrário para o 
posterior reescalonamento buscando encontrar o verdadeiro peso daquele ativo na carteira; para o PT, o que são considerados iguais são o prêmio pelo risco e a covariância dos ativos com o PT. Continuando o exemplo anterior, para os ativos

$k, n$ e $i$, então, terão que $\frac{R_{k}-r_{f}}{\sigma_{k, P T}}=\frac{R_{n}-r_{f}}{\sigma_{n, P T}}=\frac{R_{i}-r_{f}}{\sigma_{i, P T}}$. Novamente, iguala-se a expressão a um valor arbitrário, resultando em uma somatória que ultrapassa $100 \%$ e, por último, realiza-se o reescalonamento para identificar os pesos corretamente.

Com o intuito de situar o leitor, convém salientar que diversos autores têm pesquisado acerca da eficiência da otimização de média variância em relação a outras técnicas de construção de carteiras (DEMIGUEL; GARLAPPI; UPPAL, 2009, TU; ZHOU, 2011), como também estudos sobre formas alternativas à matriz de covariância amostral (LEDOIT; WOLF, 2003, PAPPAS; KIRIAKOPOULOS; KAIMAKAMIS, 2010, SANTOS; TESSARI, 2012, RUBESAM; BELTRAME, 2013), que, conforme mencionado, é necessária para o cálculo do risco segundo definido pelo autor. Por exemplo, Santos e Tessari (2012) analisaram o desempenho fora da amostra da carteira de média-variância e de mínima-variância em termos de retorno médio, desvio padrão, Índice de Sharpe (IS), turnover, custo breakeven e retorno acumulado em excesso ao Certificado de Depósito Interfinanceiro (CDI) entre cinco diferentes maneiras de calcular a matriz de covariância (matriz amostral, RiskMetrics e três estimadores alternativos propostos por Ledoit e Wolf (2003, 2004a, b), comparando os resultados com dois benchmarks: a carteira denominada ingênua $(1 / N)$ e o índice representativo da carteira de mercado (Ibovespa). Como resultado, encontraram que estimadores alternativos, com destaque para o apresentado por Ledoit e Wolf (2003), obtiveram desempenho superior inclusive em relação aos benchmarks.

\subsection{MÉTODO DE BOOTSTRAPPING E SIMULAÇÃO DE MONTE CARLO}

Assim como na literatura se tem buscado novas formas de estimar o risco, também se tem realizado estudos voltados a diferentes formas de gerar os dados para os quais retorno e risco e variáveis de input serão calculados.

Michaud e Michaud (2008) retomam uma discussão sobre a Resampled Efficient Frontier (REF) apresentada por Michaud (1998). Essa técnica busca compensar a fronteira de média-variância tradicional de Markowitz (1952) por entender que esta não considera os erros de estimação, isto é, a incerteza das variáveis que servem 
de input para estruturar a melhor relação retorno versus risco. Sobre isso, Michaud e Michaud (2008) apontam que:

[...] the problem that limits the investment value of MV optimized portfolios is not Markowitz's theory. Markowitz gives you the right way to invest given that you know that you have exactly the correct inputs. The most serious problem is estimation error, or parameter uncertainty, in optimization inputs. (MICHAUD; MICHAUD, 2008, p. 10).

Dessa forma, Michaud (1998, 2002) e Michaud e Michaud (2008) têem levantado uma discussão acerca do uso de portfólios ótimos por meio de reamostragem. A REF faz uso do método de bootstrapping e do Método de Simulação de Monte Carlo (MSMC) visando à construção de carteiras que contemplam o grau de acurácia nas informações, ou seja, que atentam para a incerteza (MICHAUD; MICHAUD, 2008, p. 10).

O bootstrapping é um método não paramétrico, o qual, a partir dos dados originais, gera pseudoamostras. Segundo Efron e Tibshirani (1986), havendo um parâmetro desconhecido, $\theta$, e dado à escolha de um estimadixor, $\theta$, para este, o bootstrap permite identificar o quão acurado o estimador escolhido, $\theta$, é diante do $\theta$. Partindo de uma amostra, $X=X_{1}, X_{2}, X_{3}, \ldots, X_{n}$ é construído um novo conjunto de dados $B=x_{1}, x_{2}, x_{3}, \ldots, x_{n}$, em que as pseudo-observações são encontradas a partir de sorteios com reposição, podendo ocorrer de uma determinada variável ser elencada na amostra de bootstrapping $(B)$ mais de uma vez (para maiores detalhes acerca do método, ver Efron (1979), Efron e Gong (1983) e Efron e Tibshirani (1986)). Já no MSMC, busca-se não o sorteio aleatório a partir dos dados já existentes, mas recriar os dados partindo de uma distribuição de probabilidade.

Em Michaud e Michaud (2008), os autores apresentam que ao contrário de outros estudos que comparam a performance in-sample da metodologia tradicional de MV com a REF, estão concentrados no que se refere ao out-of-sample, para identificar a superioridade da REF. O Gráfico 1 representa fronteiras eficientes construídas por simulação (10 anos de observações mensais) para 20 companhias escolhidas aleatoriamente a partir do índice S\&P 500 em contraste com o método tradicional de MV. 
Gráfico 1 - Fronteira Eficiente Original e Simuladas e a Fronteira Resampled Efficient (RE)
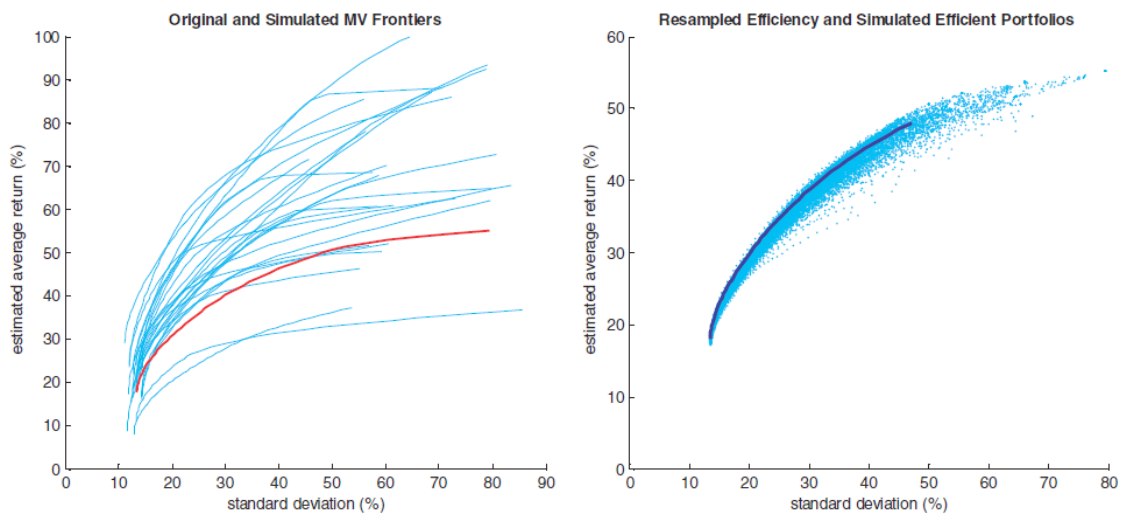

Fonte: Michaud e Michaud (2008, p. 11).

O que os autores quiseram apresentar no Gráfico 1 do lado esquerdo, é a variabilidade que ocorre quando se parte de fronteiras simuladas chegando, inclusive, a algumas apresentarem metade do risco e outras possuírem maior grau de risco. No lado direito, comentam que as várias fronteiras simuladas se convertem em uma única a partir da RE, que parte do pressuposto de que como há uma probabilidade igual de ocorrer cada uma das fronteiras simuladas, então é possível efetuar uma média dos pesos das carteiras de mínima variância, de máximo retorno e das intermediárias de forma a apreciar a utilidade esperada do investidor. Ainda, Michaud e Michaud (2008) apresentam que a RE faz uma melhor distribuição dos pesos dos ativos nas carteiras, ao contrário da tradicional que em determinadas suposições atribuem peso nulo para alguns ativos.

Dessa forma, no presente trabalho serão levantadas as fronteiras eficientes a partir do método descrito na parte de carteiras, com dados históricos, que, para simplificar, será aqui, por vezes, denominada forma tradicional por representar uma maneira bastante utilizada, por meio do método de bootstrapping e por MSMC, contudo, analisando a performance in-sample para comparação. 


\section{PROCEDIMENTOS METODOLÓGICOS}

\subsection{DELINEAMENTO DA PESQUISA}

Vergara (2000) realiza uma classificação da pesquisa em dois tipos: quanto aos fins e quanto aos meios. Assim, este artigo encontra-se enquadrado como quantitativo de método dedutivo; quanto aos fins é classificado como descritivo e quanto aos meios como ex post facto, pois se refere a metodologias alternativas para a construção da fronteira eficiente sem, contudo, haver como alterar os resultados já atingidos pelos ativos analisados.

\subsection{AMOSTRA}

Os ativos utilizados neste trabalho são American Depositary Receipts (ADRs). Segundo Assaf Neto (2009, p. 71), as companhias brasileiras podem fazer uso dos Depositary Recepeits (DRs), em português recibos de depósito, emitindo recibos de depósitos lastreados em ações, em que estas são custodiadas por uma instituição financeira denominada Banco Custodiante e, com a existência desse lastro, emitidas por um Banco Emissor. Especificamente, os ADRs são um tipo de recibo de depósito lançado nos Estados Unidos e representam uma forma de as empresas brasileiras captarem recursos no mercado americano. Convém destacar que existem ainda outros tipos de DRs consoante o mercado em que são negociados, por exemplo, o Brazilian Depositary Receipts (BDRs), empresas estrangeiras que negociam na Bolsa brasileira.

Para fins desta pesquisa, foram utilizados os ADRs classificados como Top Components do Dow Jones Brazil Titans ADR Index (BR 20) ou apenas BR Titans 20, na carteira do índice de 2013, transacionados na New York Stock Exchange (NYSE). O BR Titans 20 é um índice calculado desde outubro de 2004, pertencente à família dos Dow Jones Indexes. Assim como no caso brasileiro, tem-se o índice representativo da carteira de mercado (Ibovespa), o BR Titans 20 representa as maiores e mais líquidas ações das companhias brasileiras listadas nos Estados Unidos.

A seguir, tem-se o Quadro 1 apresentando as companhias que fazem parte da análise. 
Quadro 1 - Códigos dos ativos utilizados na pesquisa

\begin{tabular}{|l|c|}
\hline \multicolumn{1}{|c|}{ Empresa } & ADR \\
\hline Itaú Unibanco Holding S.A. & ITUB \\
\hline Banco Bradesco & BBD \\
\hline Petrobras S.A. & PBR \\
\hline Cia Bebidas das Américas - Ambev & ABV \\
\hline Vale S.A. & VALE \\
\hline BRF S.A. & BRFS \\
\hline Ultrapar Participações S.A. & UGP \\
\hline Telefônica Brasil S.A. & VIV \\
\hline Gerdau AS & GGB \\
\hline Cia Brasileira de Distribuição & CBD \\
\hline
\end{tabular}

Fonte: os autores.

Dentro dos setores para os quais as empresas pertencem, pode-se visualizar um mix abrangendo, por exemplo, o setor financeiro, alimentício e de telecomunicações. Também, apesar de aqui terem sido apresentadas apenas as 10 maiores e mais líquidas empresas, destaca-se que o índice é composto por 20 companhias brasileiras.

\subsection{TRATAMENTO DOS DADOS}

Os preços de fechamento foram coletados através do Software Economática, para, então, calcular os retornos das séries. O período compreendido nessa análise é de fevereiro de 2010 a abril de 2013 , em base mensal ( $T=39$ observações). Os retornos das séries foram calculados conforme a Equação 6.

$$
\frac{P_{t}-P_{t-1}}{P_{t-1}}
$$

Para construir a fronteira eficiente, fez-se uso das Equações 1 e 2, para obter o retorno e o risco, respectivamente, porém, sem restringir vendas a descoberto (o ativo pode assumir pesos negativos, i.e. $w<0$ ). Para encontrar o portfólio de variância mínima e tangente, baseou-se nas Equações 4 e 5. 
A partir de então, pode-se encontrar a fronteira para os dados originais e também com os retornos mensurados a partir do método de bootstrapping e pelo MSMC. O primeiro sorteia aleatoriamente e com reposição o conjunto de observações mensais a entrar no cálculo da fronteira como retornos obtidos no período. Para tanto, foi realizado tal procedimento com o número de observações equivalente ao total da amostra $(T=39)$ e com o dobro dessa janela de tempo $(T=$ 78), buscando indícios comparativos sobre a superioridade de um procedimento em relação aos demais.

Pelo MSMC, foram simuladas 1.200 observações randômicas mensais situadas no intervalo de -2 a 2 com o objetivo de compreender períodos de tempo maiores para a comparação. Mais ainda, para não perder características gerais do inter-relacionamento entre as variáveis, procedeu-se ao calculado de decomposição de Cholesky na matriz de covariância para considerar a forma como elas se movimentam conjuntamente. Sobre tal procedimento, Greene (1993, p. 36) define que " $[\ldots]$ qualquer matriz definida positiva simétrica, $A$, pode ser escrita como o produto de uma matriz triangular inferior, $L$, e sua transposta (que é uma matriz triangular superior), $L^{\prime}=U$. Assim, $A=L U$. Esta é a decomposição de Cholesky de $A$." Dessa forma, os retornos foram calculados como:

Assim, como estão sendo propostos três tipos de estratégia de investimento com quatro possíveis formas de construir um portfólio eficiente (bootstrapping com total de observações igual ao tamanho da amostra $-T=39$, bootstrapping com o dobro do total de observações da amostra $-T=78$, pelo critério tradicional e por Simulação de Monte Carlo), foi conduzido o teste t para os máximos Índices de Sharpe para verificar se estatisticamente há diferenciação no uso de uma estratégia mensurada por tal indicador sobre as demais. O Índice de Sharpe (1966) é uma das métricas utilizadas para analisar o retorno em excesso ao mercado frente ao risco, conforme a seguir: 


$$
I S=\frac{E\left(r_{p}\right)-r_{f}}{\sigma_{p}}
$$

Dessa forma, o IS é determinado pelo:

$\mathrm{E}\left(r_{p}\right)=$ retorno esperado médi da carteira;

$r_{f}=$ retorno da proxy do ativo livre de risco;

$\sigma_{k}=$ desvio padrão (risco) da carteira.

Porém, aqui será utilizado um ajuste sendo considerada apenas a relação do retorno médio da carteira pelo desvio padrão para a comparação dessa medida de desempenho ajustada ao risco. Cabe destacar que para o cômputo dos máximos Índices de Sharpe foi considerado que:

a) para as carteiras sorteadas com reposição (bootstrapping) - apurou-se o maior IS de cada fronteira e se calculou a média deles;

b) para a fronteira tradicional e de Simulação de Monte Carlo - para essas em que foram geradas apenas uma única fronteira, considerou-se o valor máximo do IS registrado.

O valor da estatística $t$ foi calculado pela razão entre a diferença do IS gerado a partir da fronteira de bootstrapping comparado à Tradicional e, posteriormente, ao MSMC, pelo desvio padrão da estratégia de bootstrapping. O que se pretende realizar é a comparação do valor da estatística t, observando as hipóteses do teste: $H_{0}=$ não há diferença entre os máximos Índices de Sharpe construídos considerando as diferentes estratégias; $H_{1}=$ há diferença entre pelo menos um dos máximos Índices de Sharpe construído considerando as diferentes estratégias.

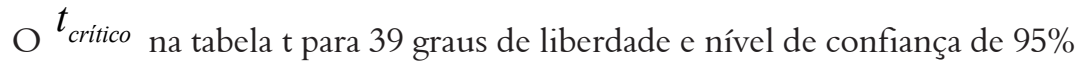
( $\alpha=0,05$ ), é de 2,02 , sobre o qual será analisado se:

$$
\left|t_{\text {calculado }}\right|<\left|t_{\text {cricito }}\right| \text { : Não se pode rejeitar } H_{0}
$$




\section{ANÁLISE E DISCUSSÃO DOS RESULTADOS}

\subsection{ESTATÍSTICA DESCRITIVA DOS DADOS}

Para iniciar a análise dos dados, convém apresentar a estatística descritiva para os 10 ADRs presentes na amostra. A seguir, segue o resultado apurado:

Tabela 1 - Estatística descritiva da amostra

\begin{tabular}{|c|c|c|c|c|c|c|c|c|c|c|}
\hline & ITUB & BBD & PBR & $\mathrm{ABV}$ & VALE & BRFS & UGP & VIV & GGB & CBD \\
\hline $\begin{array}{l}\text { Retorno } \\
\text { médio }\end{array}$ & $-0,1 \%$ & $0,68 \%$ & $-1,68 \%$ & $2,42 \%$ & $-0,64 \%$ & $1,98 \%$ & $2,53 \%$ & $1,1 \%$ & $-1,22 \%$ & $1,4 \%$ \\
\hline & $0,96 \%$ & $1,18 \%$ & $-2,98 \%$ & $1,94 \%$ & $-1,74 \%$ & $2,44 \%$ & $1,95 \%$ & $1,85 \%$ & $-2,52 \%$ & $2,56 \%$ \\
\hline $\begin{array}{l}\text { Máxi- } \\
\text { mo }\end{array}$ & $21,81 \%$ & $25,68 \%$ & $21,54 \%$ & $17,16 \%$ & $18,44 \%$ & $18,3 \%$ & $14,58 \%$ & $12,62 \%$ & $23,51 \%$ & $24,16 \%$ \\
\hline $\begin{array}{l}\text { Míni- } \\
\text { mo }\end{array}$ & $-20,08 \%$ & $-18,75 \%$ & $-25,77 \%$ & $-15,08 \%$ & $-21,4 \%$ & $-16,86 \%$ & $-11,39 \%$ & $-18,68 \%$ & $-19,26 \%$ & $-29,79 \%$ \\
\hline $\begin{array}{l}\text { Desvio } \\
\text { padrão }\end{array}$ & $9,41 \%$ & $8,37 \%$ & $11,15 \%$ & $7,66 \%$ & $9,7 \%$ & $7,9 \%$ & $6,48 \%$ & $6,5 \%$ & $10,12 \%$ & $9,89 \%$ \\
\hline $\begin{array}{l}\text { Assime- } \\
\text { tria } \\
\text { Curtose }\end{array}$ & $\begin{array}{l}0,118 \\
2992\end{array}$ & $\begin{array}{l}0,601 \\
4,496\end{array}$ & $\begin{array}{l}0,018 \\
2544\end{array}$ & $\begin{array}{r}-0,318 \\
2,927\end{array}$ & $\begin{array}{l}0,152 \\
2,509\end{array}$ & $\begin{array}{r}-0,133 \\
\end{array}$ & $-0,161$ & $\begin{array}{r}-1,295 \\
5268\end{array}$ & $\begin{array}{l}0,431 \\
2948\end{array}$ & $-0,699$ \\
\hline
\end{tabular}

Fonte: os autores.

Quanto ao retorno médio, a UGP ficou em primeiro lugar no ranking com 2,53\%, seguida da ABV e BRFS, que alcançaram 2,42 e 1,98\%, respectivamente. Os papéis ITUB, PBR, VALE e GGB foram aqueles que obtiveram retorno médio negativo durante o período de amostra. A PBR apresentou o maior desvio padrão $(11,15 \%)$ e a UGP o menor desvio em relação à média (6,48\%). Ainda, cabe aqui destacar dois pontos relevantes: a UGP foi a que obteve maior retorno e menor risco mensurado pelo desvio padrão; as companhias que tiveram retorno médio negativo estão entre aquelas que apresentaram maior desvio padrão.

Sobre a assimetria e curtose, cinco papéis mostram assimetria negativa e em geral todos evidenciam a presença de curtose, porém, de baixa magnitude considerando a comparação com o padrão da distribuição gaussiana que exibe curtose 3 .

\subsection{CONSTRUÇÃO DA FRONTEIRA EFICIENTE PELOS MÉTODOS PROPOSTOS}

O Gráfico 2 representa a fronteira eficiente segundo os critérios de média-variância levantados por Markowitz, a partir da abordagem de método de 
reamostragem por bootstrapping com a mesma quantidade de observações que na MV tradicional $(\mathrm{T}=39)$.

Gráfico 2 - Fronteira Eficiente com Bootstrap $(T=39)$ e Tradicional de Markowitz

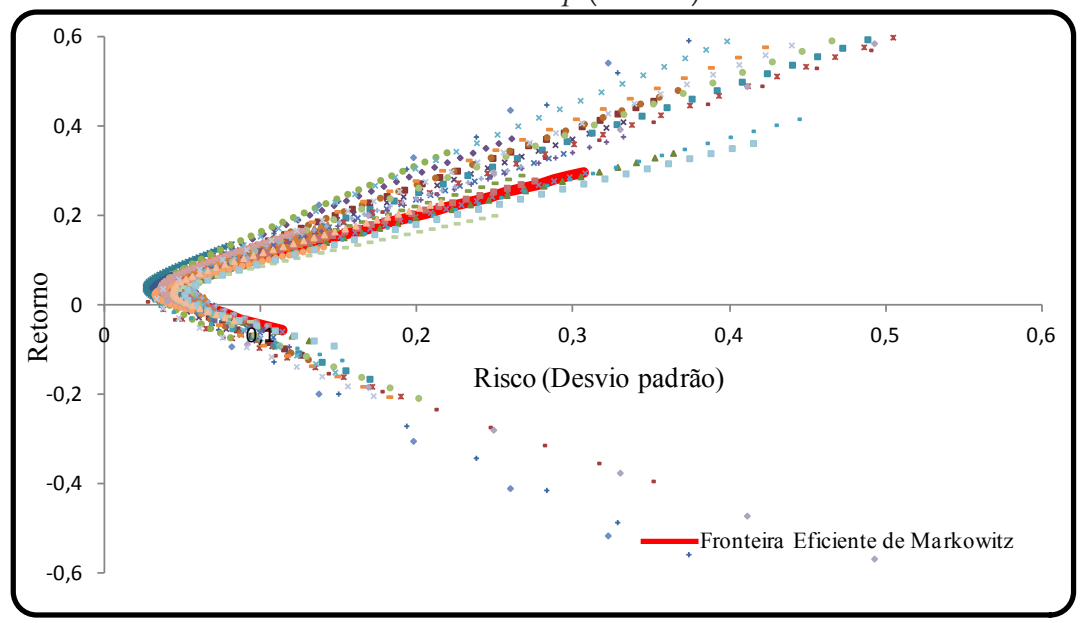

Fonte: os autores.

Porém, ainda assim o que é evidente é que, da mesma maneira que Michaud e Michaud (2008), por meio da técnica de Resampling Efficiency (RE) com o Método de Simulação de Monte Carlo, aqui também a fronteira eficiente MV tradicional foi superada havendo uma grande variabilidade com fronteiras simuladas com menor e maior risco que a tradicional. Para o resultado de bootstrapping com o dobro do número de observações $(T=78)$, também se encontrou uma superioridade observando as fronteiras simuladas. O Gráfico 3 ilustra o resultado: 


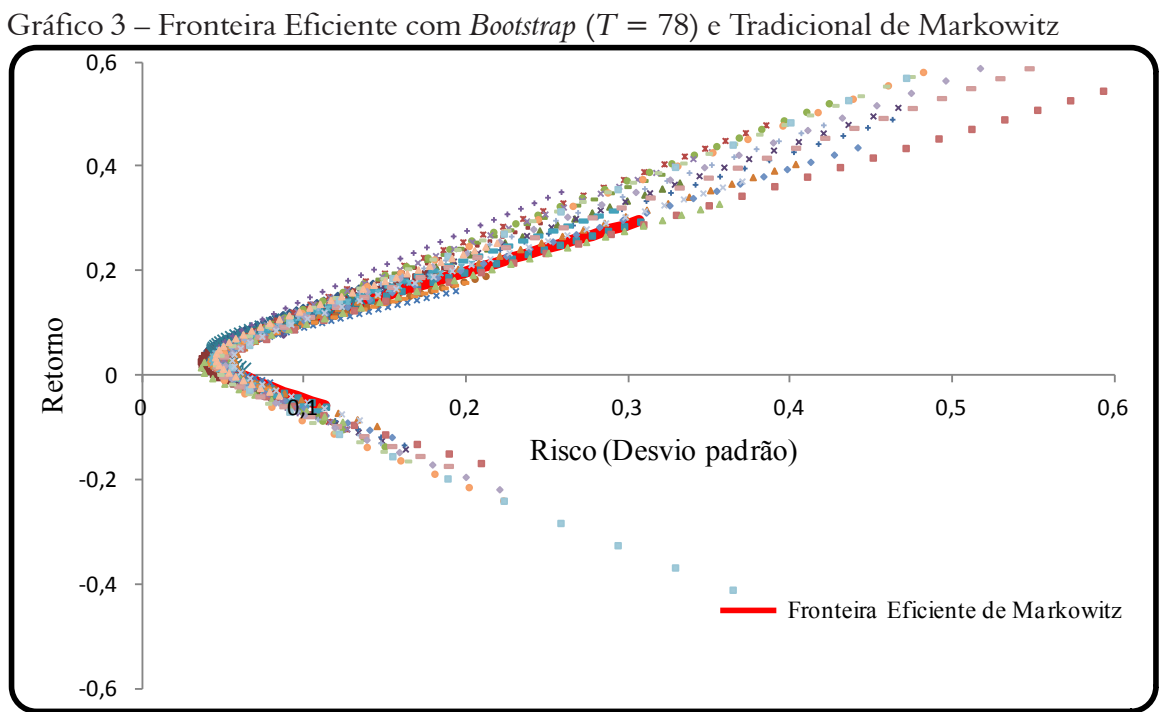

Fonte: os autores.

Logo, percebe-se que resultado similar é encontrado, existindo a possibilidade de construir fronteiras por boostrapping que estão acima e abaixo da fronteira eficiente de Markowitz (1952); porém, havendo muito mais aquelas que a superam. Buscando aprofundar a análise, realizou-se a simulação da fronteira a partir do Método de Monte Carlo para comparar com a fronteira de MV. Por Simulação de Monte Carlo foi recriado um total de 1.200 observações mensais com o objetivo de permitir um maior espaço de tempo amostral para a análise e em razão das incertezas no mercado, buscando, assim, abranger vários cenários possíveis. Aqui, optou-se por primeiramente apresentar apenas o plot da fronteira por MSMC e, posteriormente, comparada à tradicional, em decorrência do grau de retorno e risco computados que são bastante inferiores quando em contraste. 
Gráfico 4 - Fronteira Eficiente pelo Método de Simulação de Monte Carlo

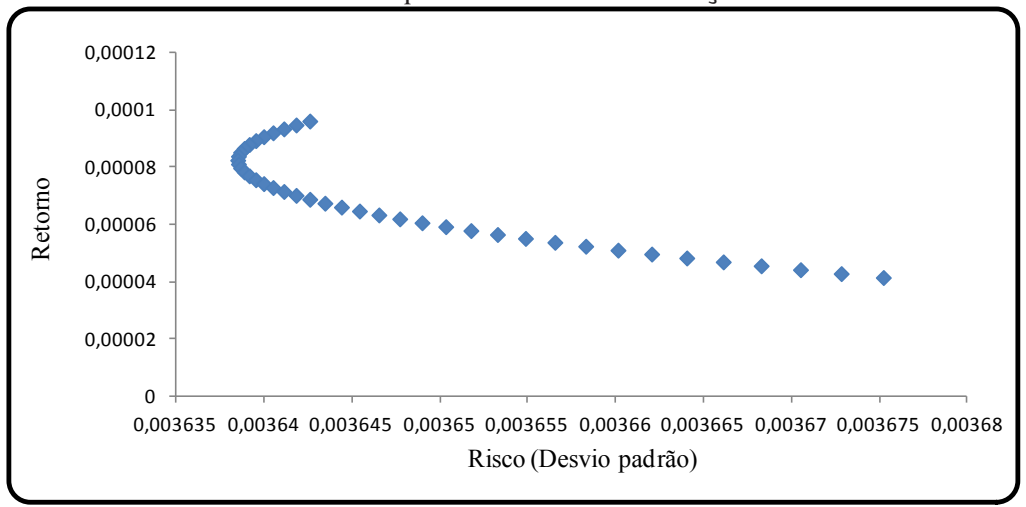

Fonte: os autores.

Observa-se no Gráfico 4 um risco praticamente constante ao longo da fronteira e um retorno também com pouca variação. Isso significa que para uma análise mensal, cobrindo 1.200 observações, o MSMC gerou uma baixa relação retorno/risco e, consequentemente, baixo IS, o que era um resultado esperado em decorrência do tamanho da série gerada. No Gráfico 5 mostra-se a comparação das duas fronteiras:

Gráfico 5 - Fronteira Eficiente com MSMC e Tradicional de Markowitz

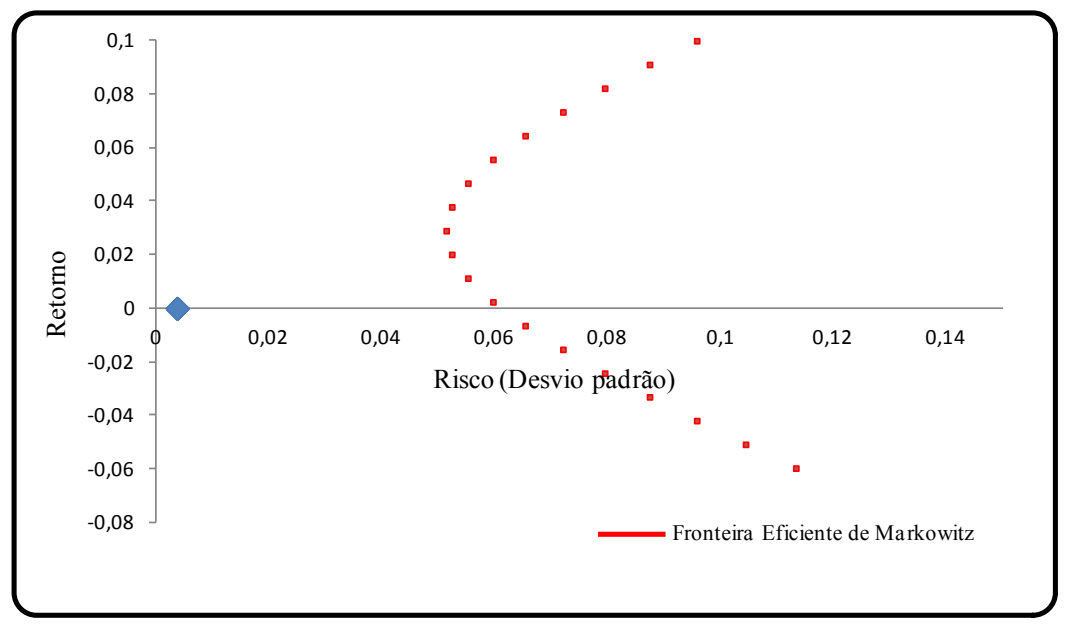

Fonte: os autores.

Com isso, verifica-se o distanciamento existente entre as duas fronteiras eficientes, o que está de acordo com o resultado apresentado no Gráfico 4. Para 
obter uma estatística de teste e verificar se realmente alguma das estratégias gerou retorno diferenciado para o investidor, foram computados os ISs. Na Tabela 2 são sintetizados os resultados referentes à estatística descritiva média e ao desvio padrão dos ISs.

Tabela 2 - Índices de Sharpe pelos diferentes métodos propostos

\begin{tabular}{lccc}
\hline IS & Bootstrap $(\mathbf{T}=\mathbf{3 9})$ & Bootstrap $(\mathbf{T}=\mathbf{7 8})$ \\
\hline Média do máximo & 1,3156 & 1,1856 \\
Desvio padrão & 0,2433 & 0,1313 \\
\hline IS & Tradicional & & MSMC \\
\hline Máximo & & 1,0444 & 0,0264 \\
Desvio padrão & 0,5289 & 0,0045 \\
\hline
\end{tabular}

Fonte: os autores.

Entre as reamostragens com reposição, o IS médio foi maior quando se fez um tamanho de amostra igual à matriz original dos dados, porém, a diferença não foi muito elevada diante da reamostragem com o dobro de observações sorteadas. Quando apenas foi construída uma única fronteira, o MSMC apresentou o menor IS que pode ser justificado considerando-se o tamanho da amostra. Agora, pode-se obter valor do teste $t_{\text {calculado }}$. Na Tabela 3 são apresentadas as estatísticas t.

Tabela 3 - Teste t calculado para os Índices de Sharpe

\begin{tabular}{lrrr}
\hline Teste $\mathbf{t}$ & Bootstrap $(\mathbf{T}=\mathbf{3 9})$ & Bootstrap $(\mathbf{T}=\mathbf{7 8})$ & \\
\hline Tradicional & 1,1194 & 1,0753 \\
MSMC & 5,3211 & 8,8277 \\
\hline
\end{tabular}

Fonte: os autores.

Observa-se que quando tais resultados são comparados com a estatística da tabela $\mathrm{t}\left({ }^{{ }_{\text {crítico }}}\right.$ ), não se pode rejeitar a hipótese nula ao nível de significância adotado (5\%) de que os máximos Índices de Sharpe calculados a partir do bootstrapping e comparados com o método Tradicional para montar a fronteira são iguais. Porém, comparando bootstrapping com a Simulação de Monte Carlo, pode-se rejeitar a hipótese nula de que são estatisticamente iguais em razão do fato de esta última metodologia (MSMC) ter gerado ISs muito baixos, o que elevou à estatística t. Duas razões que podem ter influenciado tal resultado tratam do tamanho da janela simulada que 
totalizou 1.200 retornos mensais, já que o uso da técnica é mais aconselhável para períodos de tempo maiores e, principalmente, em decorrência da forma como foi empregado, uma vez que a simulação foi construída para um intervalo de retornos mensais com variação compreendida entre -2 e 2, caracterizando a distribuição gaussiana e não partindo dos retornos efetivos das companhias ou outros tipos de distribuição de probabilidade.

\section{CONCLUSÃO}

O presente trabalho revisitou o campo da teoria de carteiras estabelecida no trabalho de Markowitz (1952) comparando a construção de fronteiras a partir dos dados históricos com outros dois métodos bastante conhecidos na literatura: o bootstrapping e a Simulação de Monte Carlo. Tais métodos são citados no trabalho de Michaud e Michaud (2008) como alternativa para a montagem da fronteira eficiente. De maneira geral, as principais contribuições deste trabalho são: retomar a questão da sensibilidade da fronteira eficiente e do Índice de Sharpe produzidos a partir das métricas utilizadas; apontar alternativas possíveis de se utilizar como dados de input para a estimação da fronteira eficiente, o que em muitos casos é crítico, dependendo da janela de informações disponíveis.

Assim, foram analisadas as diferentes métricas e testado se havia uma superioridade pelos métodos alternativos aqui elencados. Pelo ajuste puramente visual das fronteiras, percebe-se que o bootstrapping supera o método Tradicional. Porém, observando pela significância estatística fornecida pelo teste $t$, não se pode rejeitar a hipótese nula de que os máximos Índices de Sharpe calculados foram iguais. Pelo bootstrapping considerado em relação à Simulação de Monte Carlo, pode-se concluir uma diferença estatística significante entre elas. Cabe ainda destacar que, como limitações deste trabalho, foram assumidos os retornos para a Simulação de Monte Carlo a partir da distribuição gaussiana, o que gera resultados bastante diferentes quando o mecanismo probabilístico dos dados não é o mesmo daquele que aqui foi tomado como referência e que a análise do desempenho ficou restringida ao teste in-sample.

Como sugestões para trabalhos futuros, convém utilizar modelos de séries temporais, como os modelos Autoregressive Integrated Moving Average (Arima) para predizer as séries de retorno associadas a diferentes estruturas de formação da matriz de covariância e os métodos alternativos aqui empregados para comparar com os resultados outrora encontrados nesta pesquisa. 


\section{REFERÊNCIAS}

ASSAF NETO, A. Mercado financeiro. 9. ed. São Paulo: Atlas, 2009.

BERNSTEIN, P. O desafio aos deuses: a fascinante história do risco. Rio de Janeiro: Campus, 1997.

DEMIGUEL, V.; GARLAPPI, L.; UPPAL, R. Optimal versus naive diversification: how inefficient is the $1 / \mathrm{N}$ portfolio strategy? The Review of Financial Studies, v. 22, n. 5, p. 1915-1953, 2009.

DOW JONES INDEXES. Dow Jones Brazil Titans $D^{M}$ ADR Index.

Disponível em: < http://www.djindexes.com>. Acesso em: 10 maio 2013.

EFRON, B. Bootstrap methods: another look at the jackknife. The Annals of Statistics, v. 7, n. 1, p. 1-26, 1979.

EFRON, B.; GONG, G. A leisurely look at the bootstrap, the jackknife, and crossvalidation. The American Statistician, v. 37, n. 1, p. 36-48, 1983.

EFRON, B.; TIBSHIRANI, R. Bootstrap methods for standard errors, confidence intervals, and others measures of statistical accuracy. Statistical Science, v. 1, n. 1, p. 54-77, 1986.

FRIEDMAN, M. There's no such thing as a free lunch. London: Open Court Publishing Company, 1975.

GREENE, W. H. Econometric analysis. 2. ed. London: Prentice-Hall, 1993.

IQUIAPAZA, R. A.; AMARAL, H. F.; BRESSAN, A. A. Evolução da pesquisa em finanças: epistemologia, paradigma e críticas. Organizações e Sociedade, v. 16, n. 49, p. 351-370, 2009.

LEDOIT, O.; WOLF, M. Improved estimation of the covariance matrix of stock returns with an application to portfolio selection. Journal of Empirical Finance, v. 10, p. 603-621, 2003.

LEDOIT, O.; WOLF, M. Honey, I shrunk the sample covariance matrix. Journal of Portfolio Management, v.30, n.4 p. 110 - 119, 2004a.

LEDOIT, O.; WOLF, M. A well-conditioned estimator for large-dimensional covariance matrices. Journal of Multivariate Analysis, v. 88, p. 365 - 411, 2004b. 
LINTNER, J. The valuation of risk assets and the selection of risky investments in stock portfolios and capital budgets. The Review of Economics and Statistics, v. 47, n. 1, p. 13-37, 1965.

MARKOWITZ, H. Portfolio Selection. The Journal of Finance, v. 7, n. 1, p. 77-91, 1952.

MICHAUD, R. An introduction to resampled efficiency. New

Frontier Advisors's Newsletter, p. 1-6, 2002. Disponível em:<https://

www.newfrontieradvisors.com/Research/Articles/documents/

IntroductiontoResampledEffiency.pdf > . Acesso em: 12 maio 2013.

MICHAUD, R.; MICHAUD, R. Estimation error and portfolio optimization: a resampling solution. Journal of Investment Management, v. 6, n. 1, p. 8-28, 2008.

MICHAUD, R. Efficient asset management. Boston: Harvard Business School Press., 1998.

MOSSIN, J. Equilibrium in a capital asset market. Econometrica, v. 34, n. 4, p. 768-783, 1966.

PAPPAS, D.; KIRIAKOPOULOS, K.; KAIMAKAMIS, G. Optimal portfolio selection with singular covariance matrix. International Mathematical Forum, v. 5, n. 47, p. 2305-2318, 2010.

RUBESAM, A.; BELTRAME, A. L. Carteiras de variância mínima no Brasil. Revista Brasileira de Finanças, v. 11, n. 1, p. 8-118, 2013.

SANTOS, A. A. P.; TESSARI, C. Técnicas quantitativas de otimização de carteiras aplicadas ao mercado de ações brasileiro. Revista Brasileira de Finanças, v. 10, n. 3, p. 369-393, 2012.

SHARPE, W. Capital asset prices: A theory of market equilibrium under conditions of risk. The Journal of Finance, v. 19, n. 3, p. 425-442, 1964.

SHARPE, W. Mutual fund performance. Journal of Business, v. 39, n. 1, p. 119138, 1966.

TREYNOR, J. L. How to rate management of investment funds. Harvard Business Review, v. 43, n. 1, p. 63-75, 1965.

TU, J.; ZHOU, G. Markowitz meets Talmud: A combination of sophisticated and naive diversification strategies. Journal of Financial Economics, v. 99, p. 204$215,2011$. 
VERGARA, S. C. Projetos e relatórios de pesquisa em Administração. 3. ed. São Paulo: Atlas, 2000.

\section{Como citar este artigo}

ROMA, Carolina Magda da Silva; IQUIAPAZA, Robert Aldo; FERREIRA, Bruno Pérez. Aplicação da fronteira eficiente por meio das técnicas de Bootstrapping Z e Monte Carlo: uma paralelização entre BM\&FBovespa e Nyse a partir das principais ADRs brasileiras. RACE, Revista de Administração, Contabilidade e $\varangle$ Economia, Joaçaba: Ed. Unoesc, v. 14, n. 1, p. 121-142, jan./abr. 2015. Disponível em: <http://editora.unoesc.edu.br/index.php/race> . Acesso em: dia/mês/ano.

Roma, C. M. da S., Iquiapaza, R. A., \& Ferreira, B. P. (2015). Aplicação da fronteira eficiente por meio das técnicas de Bootstrapping e Monte Carlo: uma paralelização

$\overleftarrow{4}$ entre BM\&FBovespa e Nyse a partir das principais ADRs brasileiras. RACE, Revista de Administração, Contabilidade e Economia, 14(1), 121-142. Recuperado em dia/mês/ano, de http://editora.unoesc.edu.br/index.php/race 